\title{
Récits de justice et office du juge en Chine
}

\section{Hélène Piquet ${ }^{\circledR}$}

\section{Résumé}

Cet article traite du concours « Le bon juge que j'aime le plus » organisé par la Cour Populaire Suprême de Chine (CPS) en 2017. Nous expliquerons l'importance du concours et les fins véritables qu'il sert dans le contexte judiciaire chinois marqué entre autres par l'exode des juges de la profession et la campagne anti-corruption du Parti Communiste Chinois. Les dossiers des dix juges modèles lauréats seront analysés afin de dégager certaines des attentes qui pèsent sur les juges chinois et le regard de la CPS sur divers enjeux de la justice. Le concours s'inscrit dans une tradition chinoise qui a traversé les siècles, le recours aux personnages modèles.

Mots clés : Chine, juges, modèles de justice, compétition

\begin{abstract}
This paper deals with the "My favorite good judge" contest organized by the Supreme People's Court of China (SPC) in 2017. We will explain the importance of the contest and the real purposes it serves in the Chinese judicial context, which is marked by an exodus of judges from the profession and the anti-corruption campaign of the Chinese Communist Party. The files of the ten winning model judges will be analyzed to identify some of the expectations that Chinese judges and the SPC have in terms of various justice issues. The contest is part of a Chinese tradition that has spanned centuries, the use of role models.
\end{abstract}

Keywords: China, judges, justice, models, competition

La Cour Populaire Suprême (CPS) de Chine a lancé en 2017 un concours national de popularité des juges intitulé « Le bon juge que j’aime le plus » ${ }^{1}$. Il nous intéresse sous plus d'un chef. La CPS assigne deux objectifs au concours. Le premier vise à mieux faire connaître le travail des juges à la population ${ }^{2}$. Le second est énoncé dans

1 Zhongguo Fayuan Wang [Réseau chinois des cours], « Wo zui xihuan de hao faguan », [Le bon juge que j'aime le plus], https://www.chinacourt.org/article/subjectdetail/id/MzAwNEjPNYABAA\% 3D\%3D.shtm (consulté le 20 janvier 2019).

2 Ibid. L'expression chinoise est : « wei le rang shehui gong cun shenru di liaojie faguan junti » [Afin de permettre à la société de comprendre en profondeur les juges] et le reste du texte précise qu'il s'agit de comprendre leur travail (liaojie tamen de gongzuo).

Canadian Journal of Law and Society / Revue Canadienne Droit et Société, 2020,

Volume 35, no. 1, pp. 45-67. doi:10.1017/cls.2019.42 
une de ses directives. Il consacre les dix lauréats, choisis parmi trente-trois dossiers soumis par la Cour, juges modèles à l'échelle nationale, désignés collectivement comme les « juges modèles d'une nouvelle ère " ${ }^{3}$. Les dix lauréats deviennent l'objet d'études obligatoires pour tous les juges chinois ${ }^{4}$. Nous croyons que ce second objectif est plus important que le premier et c'est sur celui-ci que nous nous concentrerons. L'existence même de la directive indique que la CPS attache une très grande importance aux résultats de ce concours et qu'ils lui sont utiles. La directive constitue un document officiel, émanant de la plus haute cour du pays, à valeur contraignante pour les juges chinois ${ }^{5}$. Un modèle est par définition destiné à être imité. Il est aussi associé à la formation de types ou archétypes associés à des traits de personnalité ${ }^{6}$. Nous utilisons le mot dans ces deux sens.

Notre question est la suivante : dans un contexte marqué par un exode des juges de la profession, est-ce que les attentes de la CPS eu égard aux juges chinois, telles que révélées par les lauréats du concours, sont susceptibles de les retenir, ou, au contraire, de les détourner encore davantage du métier de juge ${ }^{7}$ ? Nous présenterons les composantes des modèles telles qu'elles ressortent des dossiers des lauréats. Ces modèles, tel que nous l'illustrerons dans notre analyse, ne sont pas de nature à favoriser la rétention des juges en poste ni le recrutement. En outre, nous croyons que ces modèles sont destinés à ajouter aux critères d'évaluation existants des juges chinois. En effet, il existe un précédent récent dans l'histoire de la CPS. Lorsque celle-ci a fait ressurgir le modèle du juge Ma Xiwu, associé à la médiation judiciaire comme méthode de résolution des conflits, les cours chinoises ont aussitôt exigé des juges qu'ils usent de la médiation et très rapidement affiché sur leurs sites des taux d'affaires résolues par celle-ci afin de montrer qu'elles remportent un très grand succès en la matière ${ }^{8}$. Sur cette base, nous croyons que la dimension d'évaluation découle, au moins implicitement, des modèles constitués par les dix lauréats. En

3 Zhongguo Fayuan Wang [Réseau chinois des cours], «Renshibu zuigaofayuan lianhe biaozhang 'wo zui xihuan de hao faguan' " [Le Ministère des ressources humaines de la CPS proclame [le concours] le bon juge que j'aime le plus], https://www.chinacourt.org/article/detail/2017/12/id/ 3100677.shtml (consulté le 5 avril 2018).

4 Zhongguo Fayuan Wang [Réseau chinois des cours], « Zui Gao Renmin Fayuan Xia Fa “Guanyu kaizhan xiang" "Wo zui xihuan de hao faguan" xuexi huodong jueding " (La CPS promulgue la «Résolution sur l'ouverture de l'activité d'étude “le bon juge que j'aime le plus" »), 10 juillet 2017, https://www.chinacourt.org/article/detail/2017/07/id/2917791.shtml (consulté le 12 novembre 2018). Les activités d'étude désignent des séminaires organisés à l'échelle du pays sur une période de plusieurs mois

$5 \quad$ Pour une explication des divers documents produits par la CPS voir le site Supreme People's Court Monitor, http://supremepeoplescourtmonitor.com (consulté le 18 février 2019).

6 Dictionnaire de français Larousse, https://www.larousse.fr/dictionnaires/francais/modèle/51916 (consulté le 2 octobre 2019).

7 Sur l'exode de la profession voir : Kwai Hang Ng et Xin He, Embedded courts. Judicial decision making in China, (Cambridge : Cambridge University Press, 2017), 51, 78-79; Tiancheng You, Faguan de gaige [La réforme des juges], (Beijing : China Legal Publishing House, 2014), 143. Il emploie l'expression "daliangliushi ", qui veut dire "à grande échelle "; Susan Finder, "Why are the Chinese judges so stressed? », 27 février 2018, https://supremepeoplescourtmonitor.com/2018/ 02/27/why-are-chinese-judges-so-stressed/ (consulté le 5 juin 2018).

8 Benjamin L. Liebman, «A Populist Threat to China's Courts? », dans Chinese Justice. Civil Dispute Resolution in Contemporary China, dir. Margaret Y. K. Woo et Mary E. Gallagher (Cambridge (USA) : Cambridge University Press, 2011), 269-313, 303. Sur Ma Xiwu, voir : Xiaoping Cong, « Ma Xiwu's Way of Judging: Villages, the Masses and Legal Construction in Revolutionary China in the 1940's », The China Journal 72 (2014): 29-52. 
outre, le fait que la CPS ait adopté les concepts et le langage du Nouveau management public (NMP), donnant lieu à la conception managériale de la justice, renforce notre argument ${ }^{9}$.

En effet, le concours de 2017 est une entreprise de transformation des juges chinois, en conformité avec les visées de la justice managériale décrites par Vigour : " la montée en puissance du registre managérial dans l'institution judiciaire contribue à la redéfinition de l'image du bon magistrat ${ }^{10}$. Ce constat s'applique à notre étude : le concours de 2017 sert à redéfinir l'image du « bon juge » chinois. Vigour poursuit en affirmant qu'il s'agit de « construire une autre représentation de la justice ${ }^{11}$. C'est précisément ce qui est en cause ici, justice désignant le système judiciaire chinois, soit les cours et les juges, acteurs importants de ce système. Dans notre étude, le mot "représentation " renvoie à l'image, bonne ou mauvaise, entretenue par la population chinoise, du système judiciaire chinois, de son fonctionnement et de ses juges. Le NMP comporte également une dimension d'évaluation, formulée en termes d'objectifs (quotas) à atteindre et de " performance " au nom de l'efficacité de l'administration concernée ${ }^{12}$. Transposée à la Chine, l'administration concernée devient le système judiciaire chinois, dont le travail est évalué à travers la figure du juge, lui-même objet d'évaluation ${ }^{13}$. C'est ici que le recours au vote du public trouve son sens. Il s'agit d'une pratique courante du PCC dans le contexte plus large de la gestion des cadres du Parti, lors de leur évaluation périodique ${ }^{14}$. La performance évaluée au regard de l'atteinte d'objectifs chiffrés fait partie de leur évaluation ${ }^{15}$. Les juges sont des cadres du Parti.

Les modèles de juges ont fait l'objet de plusieurs études dans d'autres juridictions nationales. Ainsi, François Ost a identifié trois modèles de juges, qu'il rattache, dans ses propres mots, à des "modèles de justice » en Europe ${ }^{16}$. Pour sa part, Judith Resnik a traité du modèle du " juge gestionnaire » aux États-Unis. Plus récemment, Archie Zariski et Tania Sourdin ont traité du juge

9 Sur l'adoption de la justice managériale par la CPS voir Stéphanie Balme, Chine. Les visages de la justice ordinaire. Entre faits et droit (Paris: Les Presses de Science Po, 2016), 118.

10 Catherine Vigour, " Justice : l'introduction d'une rationalité managériale comme euphémisation des enjeux politiques ", Droit et société 63-64 (2006): 425-455, 432. Voir le numéro entier consacré au sujet : Rationalité juridique vs rationalité managériale? Droit et société 82, (2013/2); Daniela Piana, Judicial Accountabilities in New Europe. From Rule of Law to Quality of Justice (Burlington : Ashgate, 2010).

11 Vigour, "Rationalité managériale », 433. Sur la notion de représentation de la justice et les études auxquelles elle donne lieu, voir : Antoine Masson et Kevin O'Connor, dir., Representations of Justice (Bruxelles : P.I.E. Peter Lang, 2007); Anick Percheron, « Représentations de la loi et de la justice chez les Français de 16 à 21 ans ", Droit et Société 19 (1991): 377-388.

12 Vigour, "Rationalité managériale », 428. Sur la mobilisation des normes du NMP par le PCC voir Maria Heimer, "The cadre responsibility system and the changing needs of the party ", dans The Chinese Communist Party in reform, dir. Kjeld Erik Broodgaard et Yongnian Zheng (Abingdon : Routledge, 2006), 122-138, 127

13 Ng et He, Embedded courts, 56.

14 Heimer, "Cadre responsibility », 128.

15 Ibid., 125.

16 François Ost, « Juge-pacificateur, juge-arbitre, juge-entraîneur. Trois modèles de justice ", dans Fonction de juger et pouvoir judiciaire, transformations et déplacements, dir. Philippe Gérard, François Ost et Michel van de Kerchove (Bruxelles : Publications des facultés universitaires SaintLouis, 1983), 1-70. 
« multifonctionnel », modèle aux multiples composantes ${ }^{17}$. Enfin, Catherine Piché a présenté le nouveau modèle de juge tel qu'il ressort des dispositions du Code de procédure civile du Québec entré en vigueur en $2016^{18}$.

Cela étant, le matériau premier sur lequel se fonde notre étude est inusité, car il est constitué des dossiers des dix lauréats. Nous le traiterons en nous inspirant du courant Droit et Littérature développé en France et aux États-Unis. Il suscite des travaux fort diversifiés, depuis l'analyse juridique des contes de fées jusqu' à l'étude de Richard Posner consacrée aux opinions judiciaires traitées comme des récits ${ }^{19}$. François Ost identifie pour sa part quatre « courants qui structurent le domaine "droit et littérature ${ }^{20}$. Il s'agit : du droit de la littérature, du droit comme littérature, du droit dans la littérature et du droit par la littérature ${ }^{21}$. Nous renvoyons le lecteur à son analyse détaillée. Le travail de Posner, qui traite les décisions des juges comme des récits, nous fournit une méthode de travail que nous adaptons.

La notion de " juge modèle " imprègne autant l'imaginaire littéraire que l'imaginaire juridique chinois ${ }^{22}$. En effet, le juge, personnage historique issu du monde du droit, nourrit l'art, l'opéra, le théâtre d'ombres et la littérature chinoise. Ainsi, le juge Bao de la dynastie Song, associé entre autres à l'intégrité, est le juge le plus connu en Chine ${ }^{23}$. Nous puisons donc également dans ce répertoire culturel chinois, et dans des études consacrées à celui-ci ${ }^{24}$.

Chaque dossier des juges lauréats sera donc traité comme un récit dont l'auteur est la CPS. Ces récits consistent, précisément, en une narration d'une ou deux affaires résolues par chaque juge lauréat. Ils nous livrent les attentes de la CPS eu égard aux qualités recherchées chez les juges chinois et au fonctionnement du système judiciaire. C'est cette lecture du concours que nous privilégions plutôt que de tenter de voir dans celui-ci les véritables préférences de la population qui a voté par voie électronique ${ }^{25}$. Nous prenons donc les dossiers des dix lauréats comme

17 Tania Sourdin et Archie Zariski, «Introduction », dans The multi-tasking judge. Comparative Judicial Resolution, dir. Tania Sourdin, Archie Zariski (Sydney : Thomson Reuters, 2013),1-20; Judith Resnik, « Managerial Judges », Faculty Scholarship Series (1982): 951, https:digitalcommons.law.yale.edu/fss_papers/951 (consulté le 18 janvier 2019).

18 Catherine Piché, «Un juge extraordinaire », Les Cahiers de droit (2016): 223-245.

19 Marine Ranouilet et Nicolas Dissaux, Il était une fois... analyse juridique des contes de fées (Paris : Dalloz, 2018) François Ost, "Droit et littérature : variété d'une approche, fécondité d'un champ ", Revue Juridique Thémis de l'Université de Montréal, 49 (2015): 2-33; Alain Quemin, « Le droit à l'épreuve de la littérature; la littérature à l'épreuve du droit », Droit et société 38 (1998): 163-171, 168.

20 Ost, « Droit et littérature », 9.

21 Ibid., 9-15.

22 Nous reprenons ici les expressions employées par François Ost. Ost, « Droit et littérature », 15.

23 Wilt L. Idema, Judge Bao and the rule of law. Eight ballad-stories from the period 1250-1450 (New Jersey : World Scientific, 2010), ix.

24 Margaret B. Wan, « Court case ballads : Popular ideals of justice in late Qing and republican China ", dans Chinese Law: Knowledge, Practice and Transformation, 1530s to 1950s, dir. Li Chen et M. Zelin (Leiden : Brill, 2014), 287-320;

25 Ce serait une toute autre démarche, hors de notre propos. Nulle part sur le site du concours il n'est fait référence à l'opinion des «électeurs ", qui se chiffrent par dizaines de millions dans chaque cas. Une étude sérieuse devrait reposer sur des entretiens avec un échantillonnage de la population, suivant des méthodes éprouvées. Or, il n'y a rien de tel ici. Pour des exemples d'études sur les représentations de la justice voir le site de l'Institut des Hautes études sur la justice : https://ihej.org/ programmes/images-et-representations-de-la-justice/ consulté le 10 septembre 2019. 
point de départ, sans chercher à reconstituer les raisons du vote de la population en leur faveur.

Plutôt que de présenter chaque lauréat de manière linéaire, nous travaillons à partir des affaires traitées par les juges ${ }^{26}$. En effet, les informations significatives résident dans les récits de ces affaires et non dans les données biographiques purement factuelles tenant sur deux ou trois lignes. Chaque récit de justice donne vie au juge lauréat et sert à mettre en valeur une ou plusieurs de ses qualités. Nous en avons identifié quatre : le juge efficace, le juge frugal, le juge dévoué et proche des justiciables et le juge pacificateur.

Les quatre qualités sont construites à partir des propos des juges cités dans les dossiers, des commentaires de la CPS, des slogans de la justice employés par la CPS, de même que des éléments du contexte politique, social et culturel. Nous utiliserons également la doctrine juridique en chinois, en français et en anglais, de même, à titre subsidiaire, que quelques instruments juridiques chinois.

L'étude des résultats d'un concours national des juges tenu en Chine depuis le début des réformes en 1978 n'a jamais été effectuée dans la doctrine en chinois, en français et en anglais ${ }^{27}$. Ce concours précis ne fait l'objet d'aucune publication. Sur les vingt affaires narrées, seules celles pertinentes pour nos fins seront résumées. Toutes les sources en chinois sont traduites par l'auteure. Après avoir traité des facteurs qui motivent les juges à quitter leur profession, nous présenterons le concours et les modèles qui en sont issus, et leurs implications pour le travail des juges.

\section{L'exode des juges}

Un regard préalable sur la situation des juges chinois s'impose afin de mieux mesurer, le cas échéant, l'impact des résultats du concours sur leur office. Le nombre de juges en Chine est passé de 211990 en 2015 à 120128 en $2017^{28}$. La section qui suit explique pourquoi.

\subsection{Un travail sujet à de multiples interférences}

La Chine compte 3559 cours réparties sur quatre niveaux, avec la CPS au sommet ${ }^{29}$. Le Parti Communiste Chinois (PCC) refuse la théorie de séparation des pouvoirs ${ }^{30}$. Il en découle, comme corollaires, le rejet du concept d'indépendance judiciaire et l'absence d'un véritable pouvoir judiciaire ${ }^{31}$. Les juges chinois sont conçus comme

26 Accessibles en Chine et hors de Chine sur le site du concours.

27 Les concours de juge sont mentionnés par Balme, Justice ordinaire. 150. Pour sa part, Carl Minzner aborde certains juges modèles dans son article : Carl F. Minzner, "China's turn against the law », American Journal of Comparative Law 9 (2001): 935-984. Toutefois, il ne parle pas des concours de juges.

28 Susan Finder, "Why are the Chinese judges so stressed? ».

29 Pour le détail de l'organisation voir : Jianfu Chen, Chinese Law : Context and Transformation, (Leiden Boston : Brill/Nijhoff, 2015), 188.

30 Qianfan Zhang, "Judicial Reform in China : an overview ", dans China's Socialist Rule of Law Reforms Under Xi Jinping, dir. John Garrick et Yan Cheng Bennett (Londres et New York : Routledge, 2016), 17-29, 18 ; Balme, Justice ordinaire, 144-145.

31 Balme, Justice ordinaire, 144. 
de simples fonctionnaires de l'État, composante de l'administration chinoise ${ }^{32}$. Les ingérences du PCC dans le processus judiciaire sont nombreuses et ont été étudiées ${ }^{33}$.

Le système judiciaire fait partie intégrante de la construction de l'État ${ }^{34}$. Il est à ce jour considéré par le PCC comme un instrument de sa légitimité et de stabilisation de l'ordre social ${ }^{35}$. Cette situation entraîne d'importantes répercussions sur le travail des juges ${ }^{36}$. En effet, leur capacité à maintenir la stabilité fait partie des critères d'évaluation traités plus loin, et cette obligation de résultats distingue le travail des juges chinois de celui des juges en Amérique du Nord ou en Europe ${ }^{37}$.

Le juge You Tiancheng a constaté, dès 2014, le phénomène de l'exode de la profession et en recense plusieurs causes ${ }^{38}$. D'abord, un déficit de valorisation, dans la société chinoise, eu égard à leur statut et à leur rémunération, qui suscite du ressentiment ${ }^{39}$. L'insuffisance des salaires versés aux juges en relation avec les coûts de la vie en Chine engendre des frustrations et ouvre la porte à la corruption ${ }^{40}$. Ensuite, l'absence de distinction entre le personnel administratif des cours et les juges banalise ces derniers ${ }^{41}$. Enfin, l'absence de dispositif pour protéger la sécurité physique des juges les inquiète. Les juges subissent des représailles qui incluent des insultes, des agressions physiques et, parfois, le meurtre ${ }^{42}$.

You mentionne également «l'interférence externe ", qui désigne les renqing, un concept multiforme. Littéralement, il s'agit des "sentiments humains ». Dans le cadre de l'office du juge, l'expression vise la compassion censée être exercée par ce dernier dans l'application du droit ${ }^{43}$. Dans un deuxième sens, plus courant, les renqing font référence aux obligations interpersonnelles ${ }^{44}$. Ils font partie d'un concept plus large et central pour la société chinoise, les guanxi, le réseau de relations d'une personne ${ }^{45}$. Au fil du temps, les renqing revêtent des connotations positives ou négatives aux yeux de la société chinoise ${ }^{46}$. Ainsi, un juge du XVIII ${ }^{\mathrm{e}}$ siècle, Liu Yong, est encensé pour sa capacité à résister aux renqing ${ }^{47}$. L'appel aux

Jean-Pierre Cabestan, Le système politique chinois (Paris : Les Presses de Science Po, 2014), 271. Cabestan, Système politique chinois, 276-277; Balme, Justice ordinaire, 141-147. Pour des sources plus anciennes voir aussi, entre autres, les travaux de Stanley B. Lubman, Randall Peerenboom. Cabestan, Système politique chinois, 271.

Ibid., 279.

Sur l'évolution générale de la situation des juges chinois depuis 1978, voir Balme, Justice ordinaire, 101-134.

$\mathrm{Ng}$ et $\mathrm{He}$, Embedded Courts, 139.

Le juge You est retenu ici en sa qualité d'observateur de longue date des cours chinoises. Il a été recruté comme juge alors qu' il était professeur de droit dans une université du Sud de la Chine. Il est également l'un des juges qui s'exprime le plus, et ses ouvrages, de même que les articles de journaux qui contiennent des entrevues avec lui, sont des sources vérifiables.

You, La réforme des juges, 143. Il emploie l'expression "daliangliushi ", qui veut dire à grande échelle.

Ibid., 146 .

Ibid., 152 .

Ibid., 145

Wan, « Court Case Ballads », 301-302.

Longwen Fu et Cheris Shun-ching Chan, « Le dilemme d'Hippocrate. Guanxi et déontologie dans le secteur des soins hospitaliers en Chine ", Perspectives Chinoises, 4 (2016): 19-28, 25.

Ibid., 21

Wan, « Court Case Ballads », 301-302.

Ibid., 301. 
renqing émane des parties, des réseaux de relations de celles-ci, des proches des juges et parfois de leurs supérieurs ${ }^{48}$. La prise en compte des renqing par le juge le conduit parfois à ignorer la règle de droit applicable afin de favoriser une partie ${ }^{49}$. Mais, surtout, cette invocation des renqing place les juges dans une position très inconfortable. Ils considèrent les renqing comme une source de pressions intenables $^{50}$. Si le juge cède à l'appel des renqing, il s'expose à perdre son poste, du moins dans le contexte de la campagne contre la corruption lancée par le président Xi Jiping en $2013^{51}$. S'il résiste, il préserve son poste mais s'expose à la réprobation du cercle des personnes qui l'ont sollicité. Les sanctions sociales peuvent aller jusqu'à une rupture des relations, perspective grave pour les juges ${ }^{52}$. L'intensité des obligations découlant des réseaux de relations distingue le cas chinois des sociétés occidentales $^{53}$. Carl Minzner expose d'autres motifs d'insatisfaction qui habitent les juges $^{54}$. Le fait de subir des évaluations, tel qu'exposé dans la section qui suit, suscite chez eux un profond malaise.

\subsection{La précarisation par le système d'évaluation}

Le système d'évaluation des juges chinois a vu le jour en $2010^{55}$. Il existait, sous une autre forme, en Chine impériale ${ }^{56}$. Il s'applique aux juges individuels et aux cours, qui font l'objet de processus distincts ${ }^{57}$. Les autorités judiciaires organisent chaque année des concours d'excellence pour les cours, visant à identifier celles possédant les meilleures pratiques en matière de qualité de la justice et de gestion d'instance ${ }^{58}$. Elles deviennent des cours modèles et l'obtention de ce statut est pour elles très important car elles peuvent entre autres obtenir plus de ressources humaines et matérielles ${ }^{59}$. En revanche, une mauvaise évaluation les pénalise financièrement. Les deux processus d'évaluation, bien que distincts, sont donc interdépendants car les juges jouent un rôle décisif dans la performance des cours.

$48 \quad \mathrm{Ng}$ et He, Embedded courts, 154

49 Ibid., 142. Cai Dingjian, Lishi yu biange. Xin Zhongguo fazhi jianshe de licheng [Histoire et changement. Le processus historique de la construction du système judiciaire en Chine nouvelle], (Beijing : Zhengfa Daxue Chubanshe-Presses de l'Université Politique et Droit, 1999), 338-339. "Faguan yu renqing " [Le juge et les renqing], Renmin Fayuan Bao (Journal des cours populaires), $1^{\text {er }}$ décembre 2013, https://www.chinacourt.org/article/detail/2013//2/id/1154050.shtml (consulté le 12 septembre 2018).

$51 \quad \mathrm{Ng}$ et He, Embedded courts, 160.

52 "Faguan yu renqing" [Le juge et les renqing].

53 Fu et Chan, « Dilemme d'Hippocrate », 28.

54 Carl Minzner, "Legal Reform in the Xi Jinping Era », Asia Policy 20, n 4 (2015): 4-9. http:// ir.lawnet.fordham.edu/faculty_scholarship/653 (consulté le 30 septembre 2019).

55 Taisu Zhang, "The pragmatic court : Reinterpreting the supreme people's court of China ", Columbia Journal of Asian Law, 25, $\mathrm{n}^{\mathrm{o}} 1$ (2012): 1-61, 28.

56 Carl Minzner, «Judicial Disciplinary Systems for Incorrectly Decided Cases: :The Imperial Chinese Heritage Lives On», dans Chinese Justice. Civil Dispute Resolution in Contemporary China, dir. M. Y. K. Woo et M. E. Gallagher (Cambridge : Cambridge University Press, 2011), 58-90.

57 Wang Yaxin et Fu Yulin, "China : Mainland. Efficiency at the Expense of Quality? ", dans Civil Litigation in China and Europe. Essays on the Role of the Judge and the Parties, dir. C.H. (Remco) Van Rhee et Fu Yulin (Dordrecht : Springer, 2014), 11-38, 33.

58 Cai Yanmin, "Case Management in China's Civil Justice System », dans Civil Litigation in China and Europe, dir. Van Rhee et Yulin (Dordrecht : Springer, 2014).

$59 \mathrm{Ng}$ et He, Embedded Courts, 56-58. 
Les critères d'évaluation des juges comprennent des récompenses et des sanctions. Ils sont répartis entre diverses directives de la CPS s'y rapportant et la Loi de République Populaire de Chine sur les juges ${ }^{60}$. D'autres critères sont énoncés dans des documents internes des cours et se cumulent avec ces diverses sources ${ }^{61}$. Les principales sanctions, de nature financière, se traduisent par des réductions de salaire, voire des amendes. La perte d'emploi est réservée aux cas les plus graves ${ }^{62}$. Les juges sont en évaluation permanente, ne possèdent aucune sécurité d'emploi ni aucun salaire garanti ${ }^{63}$. L'accent mis sur la performance des juges eu égard au nombre d'affaires traitées provient directement du $\mathrm{NMP}^{64}$. Cette dimension de l'évaluation revêt une importance cardinale pour les juges ${ }^{65}$. Elle exige d'eux une grande célérité, dans un contexte où ils doivent déployer des efforts constants pour maintenir la stabilité sociale, autre critère d'évaluation déterminant. En effet, le PCC fait du maintien de la stabilité sociale un objectif prioritaire ${ }^{66}$. La capacité des juges à maintenir celle-ci est évaluée par le nombre de contestations de leurs décisions, mais aussi par la survenance d'incidents "vicieux " minant la stabilité sociale $^{67}$. Ces derniers peuvent survenir hors de tout litige porté devant une cour, mais également, à l'occasion, de ceux-ci. Les juges veulent éviter toute forme de protestation contre leurs décisions. Aussi redoutent-ils particulièrement les contestations par la voie des pétitions car ces dernières sont qualifiées par les autorités d'atteinte à la stabilité sociale ${ }^{68}$. De même, l'appel d'une décision est dommageable pour les juges, et parfois également pour la cour en tant qu'institution ${ }^{69}$. Ces facteurs conduisent les juges à faire primer le maintien de l'harmonie sur toute autre considération et grèvent lourdement leur travail ${ }^{70}$. Ils n'hésiteront pas à mettre de côté la règle de droit applicable afin de régler l'affaire et d'imposer la paix aux parties ${ }^{71}$. Les juges considèrent toute affaire comme une source potentielle

60 People's National Congress, (Law on Judges of the People's Republic of China), disponible sur http:// en.pkulaw.cn.

61 Minzner, "Judicial Disciplinary ", 63. Ces documents internes ne sont pas accessibles aux chercheurs.

62 Law on Judges, art. 24

63 You, La réforme des juges, 145.

64 Vigour, "Rationalité managériale », 428; Daniel Mockle, « La justice, l'efficacité, l'imputabilité ", Les Cahiers de droit, 54, $\mathrm{n}^{\circ}$ 4, (2013): 613-688, 622; Balme, Justice ordinaire, 116-117.

65 Kristie Thomas, «Dynamism in China's civil procedure law: Civil justice with Chinese characteristics ", dans The Dynamism of Civil Procedure-Global Trends and Development, dir. Colin B. Picker et Guy L. Seidman (Dordrecht : Springer, 2016) 119-140, 122, 139.

66 Carl Minzner, How China's authoritarian revival is undermining its rise. End of an era, (Oxford : Oxford University Press, 2017), 97-98.

67 En chinois : exing shijian. Xin He, « No Malicious Incidents: the Concern for Stability in China's Divorce Law Practice ", (2017) RCCL Research Paper Series, accessible sur SSRN : https://ssrn. com/abstract=2914856 ou http://dx.doi.org/10.2139/ssrn.2914856, 1-22, 2 (consulté le 20 septembre 2018)

68 Ibid., 65. Sur les pétitions : Isabelle Thireau et Hua Linshan, Les ruses de la démocratie. Protester en Chine, (Paris : Seuil, 2010).

69 Minzner, «Judicial Disciplinary », 72.

70 Fu Yulin, "Social harmony and the cost to trust crisis: Goals of civil justice in China », dans Goals of Civil Justice and Civil Procedure in Contemporary Judicial Systems, dir. Alan Uzelac (Dordrecht: Springer, 2014), 167-182, 177. Les travaux de Xin He et NG le démontrent dans les cas de violence conjugale. Xin He et Kwai Hang Ng, «In the name of harmony: The erasure of domestic violence in China's judicial mediation ", International Journal of Law, Policy and the Family 27 (2013): 97-115.

71 Benjamin Liebman, "A return to populist legality? Historical legacies and legal reform ", dans Mao's invisible hand. The political foundations of adaptive governance in China, dir. Sebastian 
de troubles pour eux, plutôt qu'une occasion de faire appliquer le droit afin d'en sanctionner une violation ${ }^{72}$. Plus l'affaire est contentieuse, plus elle est susceptible d'être traitée par médiation afin d'éviter que les questions juridiques de fond, à la source du conflit, n'éclatent au grand jour ${ }^{73}$. L'évaluation et les multiples critères de celle-ci constituent sans conteste la plus grande source d'inquiétudes, au quotidien, pour les juges chinois ${ }^{74}$.

\section{Le concours de juges, outil de gestion des juges}

Le concours de 2017 trouve tout son sens si on l'envisage comme un outil de gestion des juges pour la CPS, permettant d'imposer aux juges les méthodes et comportements à embrasser dans leur travail.

Il répond à des besoins découlant de divers objectifs énoncés par le PCC. Le NMP est caractérisé par l'accent mis sur des objectifs plutôt que sur des règles et valorise la flexibilité du système ${ }^{75}$. Or, c'est un des points saillants des concours de juges. Ils sont des outils de réaction rapide aux orientations politiques du moment et, à ce titre, ils présentent un intérêt stratégique pour la CPS. À titre comparatif, précisons qu'en soi, le fait, pour une Cour de justice, d'entretenir des attentes par rapport aux juges qu'elle recrute et d'assujettir ces derniers à des standards élevés n'est pas propre à la Chine. Ces attentes sont souvent énumérées dans des appels de recrutement des juges ou dans des discours d'accueil prononcés par des juges en chef, et comprises par les juges comme devant être mises en pratique ${ }^{76}$. Dans le cas du concours de 2017, les qualités recherchées ne procèdent pas d'une liste organisée mais résident dans les récits composés par les dossiers des lauréats.

\subsection{Les attraits $d u$ concours}

Deux caractéristiques du concours, la flexibilité et l'insertion dans des références culturelles chinoises, revêtent une grande importance. La première sert directement la CPS et la seconde est conçue pour rejoindre et interpeller le public chinois.

\subsubsection{Un outil flexible}

Depuis les réformes de 1978, les concours de juges sont revenus en force ${ }^{77}$. Ils s'inscrivent dans une logique chinoise de la conception managériale de la justice évoquée. Notre vision est donc différente de celle de Balme, qui n’y voit qu'un

Heilman et Elizabeth J. Perry (Cambridge (MA) : Harvard University Press, 2011), 165-200, 175-177.

$72 \mathrm{Fu}$, « Social Harmony », 177.

73 Ibid.

74 Minzner, « Judicial Disciplinary », 88.

75 Heimer, "Cadre responsibility », 127.

76 Allocution de la Juge en Chefl'Honorable Élizabeth Corte, Palais de Justice de Québec, le 4 octobre 2016. Ce document était public à l'origine et figurait sur le site internet de la Cour du Québec. Il en a été retiré pour faire place à d'autres documents, mais conserve son caractère public. L'auteure de ce texte remercie l'Honorable Juge Pierre E. Audet, Directeur exécutif du Conseil de la Magistrature du Québec, qui nous l'a très aimablement transmis; Comité sur les nominations à la magistrature, http://www.ontariocourts.ca/ocj/fr/jpaac/competences/, consulté le 27 septembre 2019.

Balme, Justice ordinaire, 149. 


\section{Hélène Piquet}

exercice de propagande avec des « histoires montées en épingle ${ }^{78}$. Ces histoires, ou récits, méritent qu'on s'y intéresse. Les récits, avec les slogans et commentaires de la CPS, reflètent de multiples enjeux actuels de la justice chinoise vécus par les juges et vus par la CPS. La Cour a sûrement déterminé la forme des récits des dossiers des lauréats, mais elle n'a pas inventé les affaires, bien réelles, traitées par les juges ${ }^{79}$.

Les concours doivent également être mis en relation avec certains aspects de la gouvernance du PCC. Ce dernier lance régulièrement des campagnes politiques ayant divers objectifs. Celles-ci sont habituellement accompagnées de slogans et visent à imposer des orientations spécifiques à l'idéologie dans tel ou tel domaine ${ }^{80}$. Tous les organes du PCC doivent emboîter le pas et s'efforcer d'atteindre les objectifs visés par les slogans. En conséquence, tout le système judiciaire est assujetti à l'atteinte des objectifs nationaux déterminés par le régime ${ }^{81}$. L'importance réelle de ces slogans est souvent mal comprise hors de la Chine. Ils sont régulièrement intégrés dans les textes de doctrine publiés par les juristes chinois.

Dans la sphère judiciaire, les slogans génèrent de nouvelles manières de travailler qui, à leur tour, influencent le type de juge attendu dans le contexte du moment. Le slogan « envoyer le droit à la campagne " (song fa xia xiang) a généré le modèle $\mathrm{Ma} \mathrm{Xiwu}^{82}$. D'autres slogans façonnent de nouvelles exigences pour les juges. Le slogan "société harmonieuse " (hexie shehui) a servi d'impulsion au renouveau de la médiation judiciaire et extra judiciaire imposé par la CPS $^{83}$. Plus récemment, l'ancien président de la CPS mentionnait les " exigences ", pour le système judiciaire, découlant de divers slogans de la CPS comme « résoudre l'affaire et y mettre fin » (anjieshuliao). Il y est présenté comme une justification du virage vers une "justice active »(nengdong sifa), un autre slogan, dans la préface de l'ouvrage de la CPS consacré à ce sujet ${ }^{84}$. Ces slogans, loin d'être une vaine rhétorique, influencent donc directement le travail des cours et les juges les prennent très au sérieux. Le slogan du président Xi Jinping enjoint aux juges de faire en sorte que « chaque affaire décidée par les juges soit l'occasion pour les masses populaires de ressentir la justice et l'équité ${ }^{85}$. Aux termes de ce slogan, les cours portent ainsi la responsabilité de rétablir la confiance de la population en la justice, mission énoncée dans le slogan de la CPS, voulant que les juges doivent "faire coïncider les résultats juridiques avec les résultats sociaux ${ }^{86}$.

\footnotetext{
78 Ibid.
}

79 Il n'existe pas d'informations sur le processus de « formatage " des récits.

80 David Shambaugh, China's Communist Party. Atrophy and Adaptation, (Berkeley : University of California Press, 2008), 105-114.

81 Margaret Y. K. Woo, "The dynamism of China's civil litigation system ", dans Dynamism of Civil Procedure, 141-156, 149.

82 Tiancheng You, « Ma Xiwu shenpan de sifa jiazhi » (La valeur de la méthode Ma Xiwu de résolution des conflits), dans Faguan de Xunlian [La formation des juges], dir. Tiancheng You (Beijing : China Legal Publishing House, 2010), 151-156, 151.

83 Sur ce point voir Minzner, "China’s turn against the law ».

84 Zui Gao Renmin Fayuan [Cour Populaire Suprême], Dangdai Zhongguo nengdong sifa (Beijing : Zui Gao Renmin Fayuan Chubanshe (Maison d'éditions de la CPS, 2011), 2, 3. Les titres de la presque la totalité des chapitres contiennent le slogan " nengdong sifa ».

85 You, La réforme des juges, 60. En chinois : " yao nuli rang renmin cunzhong zai mei yi ge sifa anjian zhong douganshou dao gongping, zhengyi ».

86 Tiancheng You, « Yi an jieshiliao wei zuigao mudi » (Mettre fin à l'affaire est le but le plus élevé), dans La formation des juges, 118-121, 119. 
Les slogans du PCC comportent une obligation de réaction rapide de la part des institutions, astreintes à démontrer leur diligence pour les mettre en œuvre. La fréquence des concours de juges suggère qu'ils répondent à des besoins de la CPS. Leur principal attrait réside dans la flexibilité. Ils peuvent être déployés aisément au gré des impératifs des autorités, souvent changeants. Le concours est plus simple et plus malléable qu'une loi dont la modification suppose de mobiliser le corps législatif. Les modèles, assortis à des attentes spécifiques envers les juges, peuvent être crées et redéfinis rapidement et, si besoin est, relégués avec discrétion dans l'oubli.

\subsubsection{Les dossiers, récits de justice aux références culturelles chinoises}

Les concours de juges, dont celui de 2017, s'insèrent dans diverses références culturelles chinoises à travers les siècles. Tout d'abord, il fait appel à une pratique éducative - apprendre par les modèles - très ancrée dans l'histoire et la société chinoises, et étudiée par plusieurs auteurs ${ }^{87}$. Anne Cheng parle d'une « éducation par l'exemple et l'imitation de modèles plutôt que par conformité à des normes ou des principes posés a priori ${ }^{88}$. Nicolas Zufferey indique que cela fait partie intégrante des conceptions de l'apprentissage et du savoir associées au confucianisme ${ }^{89}$. Ce dernier est compris ici comme un système de pensée, au sens où Anne Cheng l'emploie : «Plus qu'un homme ou un penseur, et même plus qu'une école de pensée, Confucius représente un véritable phénomène culturel qui se confond avec le destin de toute la civilisation chinoise " ${ }^{90}$. Le Roy, traitant de l'autodiscipline confucéenne, affirme : «Les conduites, leur apprentissage et le respect des modèles forment un tout avec les visions du monde ${ }^{91}$. Sébastien Billioud et Joël Thoraval, qui travaillent sur le renouveau confucéen en Chine, ont présenté une pratique contemporaine d'apprentissage par l'exemple à propos du comportement filial attendu. ${ }^{92}$. La directive de la CPS érigeant les juges lauréats en modèles à étudier s'inscrit dans cette pratique.

Le théâtre d'ombre et les opéras chinois fournissent des sources de modèles incarnant des valeurs censées régir les rapports sociaux et familiaux tels les enfants parangons de piété filiale et les épouses chastes et vertueuses ${ }^{93}$. L'imaginaire juridique chinois est peuplé de juges célèbres de l'ère impériale, héros de pièces d'opéra, du théâtre d'ombres et de contes populaires ${ }^{94}$. Les dossiers des lauréats, qui contiennent les modèles, constituent des récits ayant pour trame principale le

87 Nicolas Zufferey, Introduction à la pensée chinoise. Pour mieux comprendre la Chine au XXI siècle (Paris : Hachette, 2008), 50; Anne Cheng, Histoire de la pensée chinoise; Gay Garland Reed, "Moral/political education in the People's Republic of China: Learning through role models ", Journal of Moral Education 24, $\mathrm{n}^{\circ}$ 2, (1995): 99-111; Sébastien Billioud et Joël Thoraval, Le Sage et le peuple. Le renouveau confucéen en Chine (Paris : CNRS Éditions, 2014), 370. Cheng, Histoire de la pensée chinoise, 76.

89 Ibid., 68.

90 Ibid., 55. Nous n'entrons pas dans le débat de la qualification du confucianisme comme religion ou philosophie.

91 Étienne Le Roy, Le jeu des lois. Une anthropologie «dynamique » du Droit, (Paris : L.G.D.J., 1999), 208.

92 Billioud et Thoraval, Le Sage et le peuple, 370.

93 Gay Garland Reed, " Moral/political education in the People's Republic of China », 100.

94 Idema, Judge Bao; Wan, «Court Case Ballads ». 
traitement des affaires par les juges. Les lecteurs voient ainsi le fonctionnement, dans les faits, du système judiciaire. La facture de ces récits de justice évoque celle des contes populaires, opéras ou des pièces du théâtre d'ombres. Ils sont rédigés dans un style vivant, contiennent des anecdotes, de même que certains propos employés par le juge et les parties à l'occasion des affaires.

Le théâtre d'ombres et l'opéra chinois, notamment, ont recours à des conventions artistiques qui contiennent divers indices sur les qualités morales des personnages. Ainsi, le public chinois sait reconnaître les archétypes à l'aide de différents détails, notamment les couleurs, qui renvoient au caractère des personnages d'opéra ${ }^{95}$. Il en va de même pour les lauréats du concours. L'organisation visuelle du concours, avec ses couleurs, ainsi que certains détails donnés par la CPS dans ses commentaires, est conçue pour rejoindre un public chinois rompu à l'art de décoder les éléments importants noyés dans un foisonnement de détails. Cependant, ces références, souvent implicites, ne sont pas généralement connues d'un public non spécialiste de la culture chinoise.

Chaque dossier comprend la photo, des données biographiques et professionnelles sommaires, ainsi que le résumé d'une ou deux affaires traitées par les juges ${ }^{96}$. Ce résumé constitue l'élément central du dossier. Cela est reflété par l'espace qu'il occupe dans la structure du dossier par rapport à la notice biographique. Les affaires sont narrées sans qualification juridique des faits. Le langage est compréhensible par tous. L'accent est mis sur certains faits et sur la manière employée par le juge afin de résoudre l'affaire. Les propos tenus par les juges revêtent une très grande importance, de même que les commentaires de la CPS qui apparaissent au début ou à la fin du dossier. Il s'agit souvent de slogans de la justice, invoqués par le juge ou la CPS ou les deux. Chaque affaire narrée se rapporte à une ou plusieurs préoccupations de la CPS concernant les juges et le fonctionnement du système judiciaire.

Outre la forme du récit, certains détails visuels transforment le concours en entreprise apparemment ludique. Ainsi les deux juges, sur la page officielle du concours, sont présentés sous la forme de personnages de bandes dessinées. Ils sont jeunes, presque des enfants. La couleur rouge, en toile de fond dominante, joue sur une double signification. Elle est d'abord la couleur associée aux réjouissances en Chine $^{97}$. Toutefois, le rouge est également la couleur associée au communisme ${ }^{98}$.

Les deux juges, de sexe masculin et de sexe féminin, sont vêtus de noir, portent une toge et des pantalons longs. Leur attribut distinctif est toutefois une cravate rouge portant l'emblème du Parti Communiste Chinois (PCC). L'image est festive, les deux personnages ayant les pieds dans des gerbes de fleurs associées aux remerciements et récompenses. Une montagne rouge à l'arrière-plan reproduit l'emblème du PCC. La figure masculine pointe le slogan « le bon juge que j'aime le plus », en guise d'invitation à voter. Le caractère ludique de l'image est tempéré par la présence de l'emblème du PCC sur la toile de fond et sur la personne même des

\footnotetext{
95 Zhao Menglin et Yan Jiqing, Masques d'opéra chinois (Beijing : Aurore, 1992), 14.

96 Chaque dossier fait entre 2 et 3 pages en format lettre US.

97 Voir la rubrique "Rouge», dans Wolfram Eberhard, Dictionnaire des symboles chinois (Paris : Seghers, 1983), 303-304.

98 Ce dernier n'est pas né en Chine mais en Europe occidentale.
} 
juges. Le décompte des votes en faveur de chaque candidat est donné dans une rubrique distincte.

La remise de prix a fait l'objet d'une cérémonie télévisée en juillet 2017, où domine la couleur rouge. La majorité des affaires ont trait aux matières civiles, avec deux affaires en droit pénal. Les juges lauréats œuvrent tant en milieu urbain qu'en milieu rural. Le Nord, le Sud, l'Est et l'Ouest de la Chine sont représentés.

\subsection{Les modèles de juges issus du concours}

Quatre modèles de juges ressortent du concours : le juge des gens ordinaires ou dévoué, le juge frugal, le juge pacificateur et le juge efficace. Cette classification, établie par l'auteure, est issue du décodage des dossiers au regard du contexte chinois de la justice dont des campagnes politiques du PCC suivant l'adaptation du courant Droit et littérature évoqué dans l'introduction. Les slogans de justice cités dans les dossiers, employés par les juges et par la CPS, le contenu des photos et les méthodes de résolution des conflits ainsi que les commentaires de clôture sur les juges ont été les constantes retenues pour examen. Cette classification n'a pas vocation à être exhaustive. Afin de limiter les notes de bas de page, nous renvoyons, pour les juges identifiés dans cette étude, au site du concours donné en référence au début de ce texte.

\subsubsection{Le "juge des gens ordinaires » ou juge dévoué}

Durant la période maoïste (1949-1976), tous les cadres du Parti, dont les juges, ont été assujettis au slogan du PCC «Au service du peuple » (wei renmin fuwu) ${ }^{99}$. L'objet du dévouement attendu des juges chinois a évolué avec le temps. Dans la Chine d'aujourd'hui, il vise à la fois le système judiciaire et les parties. Il est incarné dans la figure du « juge des gens ordinaires » (pingmin faguan), concept et slogan expliqués par le juge You ${ }^{100}$. Il a été mis de l'avant en 2008 par l'ex-président de la CPS, Wang Shengjun, qui critiquait alors le juge professionnel, associé à l'Occident et dépeint comme distant, trop éloigné des «masses " ${ }^{101}$. Cette position marquait une rupture avec la volonté de professionnalisation de la justice qui avait soustendu jusqu'alors les réformes juridiques ${ }^{102}$. Une caractéristique essentielle du juge des gens ordinaires réside dans des relations de proximité avec les justiciables ${ }^{103}$. Il doit développer l'esprit de famille par rapport aux parties, les accueillir chaleureusement, les écouter attentivement et exprimer de l'intérêt pour les moments doux amers qu'elles traversent ${ }^{104}$.

\footnotetext{
99 Shi Xian Yu, Faguan Daode Jianshe Yanjiue [Recherches sur la construction de l'éthique des juges] (Beijing : Shehui kexue chubanshe (Presses de l'Académie des Sciences Sociales), 2009), 83-84.

100 Tiancheng You, "Zuo yige pinfan de pingmin faguan " (Être un commun "juge des gens ordinaires »), dans La formation des juges, 23-27.

101 Xianchu Zhang, "Civil justice reforms with political agendas ", dans The Development of the Chinese Legal System. Change and Challenges, dir. Guanghua Yu (London : Routledge, 2011), 253271, 256. Nous substituons le terme " justiciables » au terme « masses".

102 Minzer, End of an era, 104.

103 You, « Être un commun "juge des gens ordinaires" », 25-26.

104 Ibid., 26.
} 
Dans chaque affaire, les juges viennent en aide à des parties vulnérables. La juge Qian Qi Hong est vice-présidente de la Cour Populaire du district de Daiyue, dans la ville de Taiyan au Shandong. Elle a traité une affaire dans laquelle le demandeur était une victime d'un accident de travail. La juge Qian a vite constaté que le demandeur frôlait l'indigence. Après avoir enquêté auprès de diverses banques, elle a appris que le défendeur, patron de l'accidenté, avait transféré tout son argent dans une banque à Chongqing sur un compte au nom de son fils. Elle est donc partie à Chongqing, moyennant un trajet de trente heures de train. Elle en est revenue avec la somme de 160,000 yuans. Elle a rendu une décision en faveur du demandeur, qui a reçu cette somme d'argent. Le dossier de la cour ne comporte aucun détail sur la manière dont la juge Qian a obtenu l'argent de la banque, fait pourtant très important. En revanche, il est fait état qu'au moment de l'affaire, elle était enceinte de deux mois et vivait une grossesse difficile. Lors du long trajet en train, elle a souffert d'un œdème du pied. Au mépris de sa santé, elle est allée jusqu'au bout de sa démarche afin de secourir le demandeur.

La juge Liu Chun Rong, présidente de la Cour Populaire de base du district de Bayan, à Harbin, fournit une double illustration de dévouement exemplaire au bien public. Elle a traité une affaire alors qu'elle était très malade, assignée au repos complet par son médecin. Elle est arrivée en cour le souffle court, fiévreuse et avec bouteille de liquide intraveineux. Une deuxième affaire l'a conduite à renoncer à son souper du Nouvel an chinois, lequel constitue le repas le plus important en Chine. Elle a traité l'affaire sur le champ, tout en veillant à faire nourrir la plaignante de quatre-vingt-deux ans. Pour sa part, le juge Zou Lai Shui, président de la Cour populaire de Lutian dans le district du lac Poyang de la province du Jiangxi, n'hésite pas à sacrifier ses jours de congé afin d'entendre des affaires. Le juge Chen Shao Hua a donné de sa poche la somme de 500 yuans au demandeur dans une affaire. Il a refusé d'être remboursé.

Une autre dimension du dévouement réside dans un souci d'accès à la justice chez le juge des gens ordinaires ${ }^{105}$. La Chine connaît une pénurie de ressources juridiques dans le monde rural ${ }^{106}$. Dans une acception plus large que le sens premier des recours aux tribunaux, l'accès à la justice englobe des initiatives de vulgarisation du droit au sein de la population par divers moyens et la possibilité, pour les justiciables, d'exprimer leurs opinions sur des projets de loi, ou d'autres mesures ayant pour objet l'appropriation du droit par la population ${ }^{107}$. Le juge des gens ordinaires répond à plusieurs de ces moyens. Le juge Zou Lai Shui crée des cours itinérantes, fait siéger la cour les jours de congés et, comme la juge Qian Ji Hong, au besoin dans les champs. Les cours itinérantes favorisent l'accès à la justice dans son sens premier, en déplaçant la cour au lieu où se trouvent les justiciables, leur épargnant ainsi de coûteux et difficiles déplacements.

La juge Cui Yu Fang, présidente adjointe de la chambre de supervision du processus judiciaire du tribunal militaire de l'Est au Xinjiang, répond

105 Ibid., 27.

106 Margaret Y. K. Woo "Law's location in China's countryside», Wisconsin International Law Journal, 29 (2011): 416-445, 428.

107 Yash Ghai et Jill Cottrell, « The rule of law and access to justice », dans Marginalized Communities and Access to Justice, dir. Yash Ghai et Jill Cottrell (New York: Routledge-Cavendish, 2010), 1-23, 3. 
quotidiennement aux questions des justiciables sur son site internet, "Xiao Cui Shuo Fa », soit " Petite Cui parle de droit ». En chinois, l'épithète " petit » précédant le nom est considéré comme une marque d'affection. Il connote aussi un rapport de proximité avec la personne concernée. Le nom de la plate-forme ne peut manquer de créer un sentiment de rapprochement entre la juge et les justiciables.

Le juge des gens ordinaires participe également à l'éducation juridique de la population en diffusant le droit en monde rural, suivant le slogan "song fa xia xiang ${ }^{108}$. Ainsi la juge Lin Mei Ying a parcouru à cette fin, depuis le début de sa carrière, plus de 5000 kilomètres. Le juge des gens ordinaires ou dévoué est apparu dans un contexte de populisme judiciaire décrit par Liebman ${ }^{109}$. Il n'est pas sans évoquer le « fonctionnaire père et mère de tous » (fumuguan) de l'ère impériale, en particulier le magistrat de district censé être bienveillant envers ses administrés ${ }^{110}$.

\subsubsection{Le juge frugal}

La résurgence d'un idéal de frugalité s'explique par la campagne anti-corruption initiée par le président Xi en 2013. L'article 8 de la directive en huit points de 2012 en illustre l'esprit: "Leaders must practice thrift and strictly follow relevant regulations on accommodation and cars ${ }^{111}$.

Des vêtements fastueux suggèrent une richesse mal acquise, association problématique dans le contexte de la lutte anti-corruption ( fan fubai). La population chinoise sait décoder les enjeux livrés par les photos et descriptions des lauréats. $\mathrm{M}^{\mathrm{me}}$ la juge $\mathrm{Gu}$ Li Mire porte de simples chaussures de sport très usées. Le juge Zou Lai Shui circule en chaloupe non motorisée et à vélo. En outre, il peut composer avec l'isolement dans des districts éloignés, ce qui est souligné positivement par la CPS.

Le juge frugal résonne dans la longue durée, révélant une préoccupation des dirigeants, alors les empereurs, envers l'intégrité des fonctionnaires de l'administration. Cette valeur est incarnée par le modèle " fonctionnaire lettré pur » ou qingguan $^{112}$. La modestie des tenues vestimentaires et du train de vie des juges est garante d'intégrité, en conformité avec certains juges modèles de l'ère impériale, tel le juge Liu, bien connu pour circuler en haillons. Ce choix vestimentaire délibéré indiquait qu'il vivait honnêtement, sans se laisser corrompre par divers pots de vins qui lui auraient fourni les moyens de se vêtir richement ${ }^{113}$.

La corruption des juges constituait déjà un problème au début des réformes ${ }^{114}$. L'intégrité est prise en compte par les autorités qui sanctionnent durement les cas

108 Zhu Suli, Song Fa Xia Xiang : Zhongguo jiceng sifa zhidu yanjiu [Faire descendre le droit vers les campagnes. Recherche sur le système judiciaire de base], (Beijing : Beiijng Daxue Chubanshe (Presses de l'Université de Beijing), 2000).

109 Liebman, " Populist Legality? ", 165-200.

110 Deborah Cao, Chinese Language in Law. Code Red (Boulder : Lexington books, 2018), 43-44.

111 Comité central du Parti communiste chinois, " Eight-point regulation », 5 décembre 2012, http:// cpcchina.chinadaily.com.cn/2012-12/05/content_15992256.htm (consulté le 19 mars 2019).

112 Idem, Judge Bao, x.

113 Wan, "Court Case Ballads », 303-304.

114 Lü Xiaobo, Cadres and Corruption. The Organizational Involution of the Chinese Communist Party, (Stanford : Stanford University Press, 2000), 191; Cai, Lishi yu biange (Histoire et changement), 332. 
de corruption ${ }^{115}$. Les Chinois sont très sensibles aux signes de richesse arborés par les cadres du Parti. Certains internautes usagers des réseaux sociaux chinois sont prompts à prendre en photo, à leur insu, des fonctionnaires portant des vêtements et accessoires de marque, indicateurs d'une aisance matérielle suspecte au regard de leurs salaires ${ }^{116}$. Ils diffusent ensuite ces images sur les réseaux sociaux avec pour but de mettre en évidence la personne concernée à des fins de condamnation publique. Cette démarche s'appelle utiliser "l'engin de recherche de chair humaine ${ }^{117}$.

La corruption des juges, réelle ou apparente, est un facteur important de l'érosion de la confiance de la population chinoise envers la justice ${ }^{118}$. Certaines photos des lauréats du concours de 2017 permettent aux autorités et aux juges de démontrer le sérieux de la lutte anti-corruption. Les détails donnés redorent le blason des juges et du PCC. Si une image vaut mille mots, les chaussures de sport élimées et la chaloupe non motorisée sont infiniment plus susceptibles d'atteindre leurs cibles qu'une énième déclaration des autorités contre la corruption proclamée dans les médias officiels. Ces références visuelles à la frugalité des juges du concours servent également une autre fin. Elles indiquent clairement à l'ensemble des juges qu'ils ne doivent espérer aucun bénéfice matériel de leur office.

\subsubsection{Le juge pacificateur}

Les deux modèles qui suivent, soit le juge pacificateur et le juge efficace, se rattachent au cadre de la gestion d'instance. La figure du juge pacificateur est bien connue dans les pays de common law et certaines juridictions de droit civil, avec l'accent mis sur la médiation judiciaire ${ }^{119}$. Cependant, elle s'actualise en fonction de paramètres très différents en Chine, dont le caractère autoritaire du régime. Si le juge chinois est également un juge gestionnaire, une partie de ses responsabilités diffèrent grandement de celles identifiées par Resnik dans le contexte américain ${ }^{120}$. Aussi la transposition de ces catégories a priori connues dans le contexte chinois doit-elle être effectuée en gardant à l'esprit ces considérations.

Le juge pacificateur est médiateur, associé au slogan de la société harmonieuse. Pour la CPS, le meilleur moyen d'atteindre l'harmonie est la médiation ${ }^{121}$. La CPS l'a présentée comme un mode essentiellement chinois de résolution des conflits, soit un paradigme local ${ }^{122}$. Les juges chinois usent abondamment de la médiation.

\footnotetext{
$115 \mathrm{Ng}$ et He, Embedded courts, 160.

116 Li Gao et James Stanyer, " Hunting corrupt officials online: The human flesh search engine and the search for justice in China ", Information Communication and Society 17, n 7 (2014): 814-829, https://dspace.lboro.ac.uk/2134/24096 (consulté le 22 janvier 2019).

117 BBC, Celia Hatton, 28 janvier 2014 "China's Internet vigilantes and the "human flesh search engine" ", https://www.bbc.com/news/magazine-25913472 (consulté le 25 janvier 2019). Les résultats de ces démarches varient.

118 Wang et $\mathrm{Fu}$, « Efficiency », 32.

119 Sourdin et Zariski, «Introduction », 1-20, 2.

120 Resnik, « Managerial Judges ».

121 Woo, "Litigation System », 150.

122 Hélène Piquet, «Les réformes juridiques chinoises diluées dans l'harmonie», Droit et société 84 (2013) : 453-473, 463.
} 
La réussite ou l'échec dans leur mission de pacification des parties touche directement l'évaluation des juges. Tel que vu précédemment, il s'agit d'abord pour eux d'éviter toute forme de contestation de leurs décisions, ou des solutions qu'ils proposent ${ }^{123}$. Ainsi, le fait de parvenir ou non à rallier les parties à une solution est pour eux d'importance capitale, tel qu'il ressort du slogan enjoignant « que les décisions de justice produisent une intégration des effets juridiques et des effets sociaux $" 124$.

Si le rôle pacificateur du juge chinois est bien documenté, les techniques par lesquelles il parvient à pacifier les parties le sont moins. Or, certains dossiers des lauréats permettent de combler ce manque d'informations. Il s'agit de la « construction de situation » telle qu'utilisée par Sun Liping, qui met en jeu l'usage de normes extra-juridiques destinées à résoudre le conflit ${ }^{125}$. Nous transposons la grille de Sun aux juges et renvoyons le lecteur à son analyse détaillée. Essentiellement, il s'agit pour le juge, anticipant qu'il se heurtera à la résistance des justiciables face à la solution qu'il propose, de les y rallier. Pour ce faire, il doit trouver le registre sur lequel jouer afin de dénouer l'impasse et d'amener les justiciables à s'acquitter de leurs obligations, sachant que l'invocation seule de la loi ne les convaincra pas. La construction de situation exige qu'il reformule le conflit qui oppose les parties en créant une situation nouvelle, de manière à les mettre en défaut eu égard à des obligations fondées sur le sens du juste qui s'applique dans les circonstances ${ }^{126}$. C'est le passage stratégique vers la contrainte de situation. Le contrôle de la formulation du conflit revêt une importance cruciale pour le juge. Cela lui donne le pouvoir informel d'amener les justiciables sur un terrain où il possède de bonnes chances de gagner ${ }^{127}$.

Sun mentionne plusieurs normes extra-juridiques invoquées par les cadres à la source de son analyse. L'une d'elles est l'adage « en accord avec les sentiments, en accord avec le sens de ce qui est juste et raisonnable, en accord avec le droit » (heqing, heli, hefa). Son intérêt réside en ce que "les gens jugent d'une action non pas d'après son contenu intrinsèque ou sa conformité aux règles officielles, mais selon l'identité de la personne qui la réalise et sa mobilisation à divers principes jugés adéquats à la situation et aux relations qu'elle englobe ${ }^{128}$. En d'autres termes, l'adage fait appel à des sanctions sociales, ressenties comme plus persuasives, et plus redoutables, que la sanction du droit ${ }^{129}$. Le juge You témoigne de la présence continue de l'adage comme norme d'évaluation de la justice d'une action dans la société chinoise des réformes ${ }^{130}$. Il a appelé ouvertement les juges chinois à user de

\footnotetext{
123 Benjamin L. Liebman, « Populist threat? », 270.

124 L'expression en chinois est : " panjue shixian le falu xiaoguo yu shehui xiaoguo de youji tongyi », Tiancheng You, « Mettre fin à l'affaire est le but le plus élevé »] 119.

125 Sun Liping, "Les armes faibles des forts. L'usage des normes informelles dans l'exercice du pouvoir », dans Disputes au villages chinois. Formes du juste et recomposition des espaces normatifs, dir. I. Thireau et H. Wang (Paris :Éditions de la Maison des sciences de l'homme, 2001), 249-286, 262.

126 Ibid., 262.

127 Ibid.

128 Ibid., 280

129 Ibid., 73.

130 Tiancheng You, "Ma Xiwu Shenpan de Sifa Jiazhi » (La valeur de la méthode Ma Xiwu de résolution des conflits », 154.
} 
heqing, heli, hefa dans leur travail ${ }^{131}$. La construction et la contrainte de situation ne peuvent être déployées efficacement que dans un contexte où les parties sont sensibles aux sanctions sociales découlant du comportement qui leur est reproché.

L'affaire des pierres tombales en fournit une illustration. Elle a été traitée par $\mathrm{M}^{\mathrm{me}}$ la juge Lin Mei Ying, présidente de la chambre de la protection des ressources aquifères de la Cour Populaire du district de Panlong de la ville de Kunming, au Yunnan.

Elle a opposé deux hommes, non reliés par la parenté, à propos de l'inscription figurant sur une pierre tombale. Chacun affirmait que la pierre tombale était celle érigée pour sa mère. Lors de la fête du nettoyage des tombes (Qingming jie), le demandeur Tian a constaté que la pierre de la tombe de sa mère était brisée et l'inscription de son nom effacée. Il a noté l'existence d'une nouvelle pierre tombale avec le nom d'une autre femme (Zhao). Il a demandé à Zhao de rectifier la situation et celui-ci a refusé. Les deux parties ont plusieurs fois saisi le comité des villageois, mais ce dernier n'a pu résoudre le conflit. Ce dernier s'est envenimé et Tian a saisi la cour. Lors d'une séance de médiation avec les parties, la juge Yin a dit: «les morts nous ont quittés et les vivants sont dans une situation déplorable. Les pierres tombales nous permettent d'honorer la mémoire des êtres chers, il ne faut pas perturber les ancêtres. Peu importe qui est dans la tombe, il y a tant de petits enfants qui viennent l'honorer, où est le mal? Que chaque partie réfléchisse à cela ». Tian a retiré sa plainte contre Zhao et l'affaire s'est réglée. Le discours de la juge Yin aux parties s'appuie sur le concept de piété filiale, xiao, dont l'une des composantes consiste à honorer les parents défunts ${ }^{132}$. Ces rites sont encore pratiqués en Chine à grande échelle et, en particulier, mais non uniquement, dans le monde rural.

Le conflit a été présenté par la juge comme un manquement à la piété filiale, car il portait atteinte à la paix des défunts. Cette construction de situation a mis les deux parties en défaut. Les propos de la juge se terminent avec l'expression d'une approbation face au fait que les deux parties honorent également la tombe de leurs parents. Ces propos valorisent le comportement des deux parties, transformées en deux personnes vertueuses et filiales. La question rhétorique de la juge Lin, « où est le mal », place les deux parties dans une contrainte de situation car le problème est formulé en termes tels qu'elles ne peuvent que mettre un terme à leur conflit. Au regard du respect de la piété filiale, la norme mobilisée, le conflit ne revêt plus de légitimité. L'on peut aussi déceler dans cette affaire une référence implicite à l'adage « heqing, heli, hefa ». Persister dans le conflit est également déraisonnable, car cela n'est plus en conformité avec le principe social partagé « heli » de l'adage. La juge a fait appel à la construction de situation du déraisonnable poussé à l'extrême ${ }^{133}$.

L'autre intérêt de l'affaire réside dans le fait que la juge Lin, après plusieurs séances de médiation avec les parties, a évalué que leur conflit présentait un risque élevé d'escalade et les en a avisées. Cette mention n'est pas anodine. Elle reflète la

\footnotetext{
131 Tiancheng You, «Shixian qing, li, fa de jiehe » (Réaliser la combinaison de qing, li et fa), 3 avril 2017, http://www.cssn.cn/fx/201704/t20170403_3475209.shtml. C'est le site de l'Académie des Sciences Sociales de Chine (consulté le 15 août 2018).

132 Billioud et Thoraval, Le Sage et le peuple, 368.

133 Sun « Les armes faibles... ", 270.
} 
grande préoccupation des autorités envers les risques d'escalade de conflits en apparence bénins $^{134}$. En l'espèce, les risques d'un conflit à caractère collectif étaient grands car tout le village était susceptible de prendre parti, soit pour Zhao, soit pour $\operatorname{Tian}^{135}$. De là à un épisode de vengeance lignagère impliquant tout le village, il n'y a qu'un pas ${ }^{136}$. L'affaire illustre comment les juges sont appelés à participer directement au maintien de la stabilité ${ }^{137}$. Elle témoigne aussi d'une autre dimension du juge pacificateur, sa capacité de réagir rapidement à des situations de crise afin de les désamorcer.

\subsubsection{Le juge efficace}

L'efficacité est issue du NMP transposé dans le domaine de justice ${ }^{138}$. Elle comporte entre autres l'obligation d'atteindre des résultats quantifiables, dont le nombre est fixé par les autorités pertinentes ${ }^{139}$. Dans les résultats du concours, la CPS indique, pour chaque lauréat, le nombre d'affaires traitées. Les cours le font déjà et attachent une grande importance à cet indicateur de performance des juges. Le nombre de dossiers traités par chaque juge est diffusé au sein des cours. Les juges qui ne répondent pas aux attentes éprouvent le sentiment de perdre la face, en plus de se sentir menacés dans leur poste ${ }^{140}$. Le juge Zou Lai Shui, qui traite en moyenne 800 affaires par an, affiche un taux de résolution des affaires de 100 pour cent. De même, l'efficacité se traduit par le fait de vider l'arriéré des dossiers de la cour (diaoche). Le juge Zou est encore cité en exemple, avec un taux de 82,5 pour cent. Une autre dimension de l'efficacité des juges chinois réside dans l'exécution des jugements. Il ne suffit pas d'avoir gain de cause, encore faut-il que la partie gagnante puisse toucher son dû. En Chine, l'exécution des jugements fait problème ${ }^{141}$. La responsabilité de l'exécution des jugements revient en pratique au juge, contrairement à la situation dans la plupart des juridictions en Occident ${ }^{142}$. Le juge Chen Shao Hua est louangé pour sa capacité à exécuter les jugements. Il organise à cette fin des campagnes d'exécution des jugements comparées à des tempêtes (zhixing fengbao) ou au tonnerre du printemps (chunlei xindong).

La capacité du juge à trouver la preuve irréfutable permettant de confondre les défendeurs et d'imposer une solution incontestable est une autre manifestation de son efficacité. Le dossier du juge Chen Chang Yi, président de la Cour Populaire de Zhongguancun, dans le district de Haidian à Bejing, en témoigne. Des travailleurs migrants chinois (mingong) à l'emploi d'un restaurant, face à un refus de

134 Liebman, « Populist threat », 270.

135 Le film «Le dernier voyage du juge Feng », réalisé en 2007, illustre que les conflits du monde rural entourant des rites et croyances peuvent facilement dégénérer et doivent être pris très au sérieux par les juges.

136 Lucien Bianco, Jacqueries et révolutions dans la Chine du XX $X^{e}$ siècle (Paris : La Martinière, 2005), 336. Il s'agit de l'obligation de venger les membres du clan.

137 Zhang, "Civil justice reforms with political agendas ", 259-260.

138 Balme, Justice ordinaire, 116; Piana, Judicial accountabilities, 30; Mockle, « La justice, l'efficacité, l'imputabilité », 625.

139 Zhang, "The pragmatic court ", 29; Thomas, "Dynamism in China's civil procedure law », 135; Heimer, « Cadre responsibility », 125.

$140 \mathrm{Ng}$ et He, Embedded courts, 57.

141 Balme, Justice ordinaire, 184.

142 Ibid., 185. 
l'employeur de leur verser leur salaire, ont saisi la cour. Bien que convaincu du bienfondé de la réclamation des mingong, le juge Chen ne disposait d'aucune preuve lui permettant de leur donner gain de cause. Il a décidé sur le champ d'aller perquisitionner en personne au siège de la compagnie de gestion où il a trouvé la preuve manquante. Il a rendu sa décision en faveur des mingong deux jours après. Quelques jours plus tard, les huit mingong ont apporté au juge une bannière en soie avec l'inscription : " traite les affaires avec justice (impartialité) et jouit du soutien du peuple ». Le juge Chen se rapproche ainsi du juge Bao, incorruptible certes, mais également perspicace, veillant à ce que les coupables soient toujours démasqués et châtiés indépendamment du rang qu'ils occupent dans la société ${ }^{143}$. Une telle affaire ne peut que rallier la population chinoise et satisfaire la volonté des autorités de voir les résultats juridiques répondre aux attentes des justiciables.

L'accent mis sur l'efficacité du juge est, comme le soulignent des juristes chinois, une arme à double tranchant ${ }^{144}$. En effet, les juges ont trois mois pour traiter une affaire s'il s'agit de la procédure sommaire, et six mois dans le cadre de la procédure ordinaire ${ }^{145}$. La célérité de la justice chinoise évite ainsi que les parties connaissent les inquiétudes liées aux délais judiciaires dans plusieurs juridictions occidentales. Toutefois, les parties risquent de se trouver face à une justice si rapide qu'elle en devient expéditive ${ }^{146}$. Dans ce dernier cas, cela suscite dans la population une méfiance envers la justice ${ }^{147}$. En outre, comme l'efficacité est l'un des critères pris en compte dans l'évaluation des "cours modèles ", ces dernières risquent de lui accorder préséance sur le respect des règles de procédure ${ }^{148}$. Les juges déplorent cette situation ${ }^{149}$.

\section{Conclusion}

La préoccupation quant aux qualités attendues des juges n'est pas propre à la Chine. Elle est exprimée, notamment en Europe et dans le monde anglo-américain, dans divers contextes et instruments juridiques, dont des codes de déontologie où ces qualités sont expressément énumérées. Toutefois, ni les cours européennes ni les cours en Amérique du Nord n'organisent de concours de popularité des juges. Le concours « Le bon juge que j'aime le plus » tenu en 2017 constitue un véhicule particulier de la CPS pour formuler ses attentes envers les juges chinois.

Les dossiers des lauréats se lisent comme de véritables récits dont les juges sont les héros. Traiter les dossiers comme des pièces du théâtre d'ombres ou d'opéra chinois s'est révélé pour nous une approche fructueuse. Nous croyons que la CPS s'est délibérément inspirée de ces formats pour engager le public dans une entreprise très importante pour elle à titre d'institution judiciaire. Le public chinois aura su identifier les détails qui comptent véritablement, comme des chaussures usées, le

\footnotetext{
143 Idem, Judge Bao, ix.

144 Wang et $\mathrm{Fu}$, « China : Mainland », 31

$145 \mathrm{Fu}$, "Social harmony », 175.

146 Cai, « Case management », 55.

147 Ibid.

148 Wang et $\mathrm{Fu}$, « China : Mainland », 33.

149 Cai, « Case management », 54.
} 
recours à un véhicule non motorisé, ou l'appréciation positive de la CPS envers la capacité de certains lauréats à vivre modestement. De tels détails risquaient d'échapper à un auditoire occidental qui n'est pas au fait de certaines conventions artistiques chinoises et du contexte chinois plus large.

Le concours s'inscrit ainsi simultanément dans le paradigme ancien d'apprendre par l'exemple ou à partir de modèles dans les pratiques de gestion et d'évaluation des cadres par le PCC de même que dans le courant du NMP. L'importance que lui attache la CPS ressort de la directive qui impose aux juges chinois l'étude des dix lauréats consacrés juges modèles d'une nouvelle ère. La CPS s'adresse aussi à la population chinoise, à qui elle veut montrer des juges au comportement irréprochable.

Les documents du concours ne livrent aucun indice permettant d'affirmer qu'un des modèles identifiés aurait préséance. Nous croyons qu'ils se cumulent, mais que certains modèles pèsent plus lourd. Les conclusions formulées ici se fondent donc sur le contexte plus large. Le modèle du juge pacificateur est destiné à occuper une position dominante durable, en raison du caractère prioritaire attaché par le PCC au maintien de la stabilité sociale comme objectif à respecter par toute l'administration publique chinoise ${ }^{150}$. Le modèle du juge frugal revêt depuis 2013 une très grande importance en raison de la campagne anti-corruption. Ces deux modèles sont fonction d'enjeux politiques sensibles qui touchent potentiellement à la légitimité des dirigeants. Le juge efficace et le juge des gens ordinaires et dévoué reflètent des préoccupations importantes, certes, mais moins sensibles. L'efficacité comme norme de fonctionnement du système judiciaire est valorisée dans plusieurs juridictions à travers le monde. Le modèle du juge efficace est donc transversal et témoigne de l'absorption, par les autorités judiciaires chinoises, de certains concepts du NMP. Le juge des gens ordinaires et dévoué illustre une tentative de création d'un modèle censément adapté aux besoins de la Chine. Si l'hostilité officielle contre les "modèles occidentaux " s'atténue, ce modèle revêtira sans doute moins d'importance ${ }^{151}$. L'intégrité, la capacité à maintenir la stabilité et l'efficacité constituent déjà des critères d'évaluation par les cours aux termes de la Loi de République Populaire de Chine sur les juges. Le concours s'inscrit donc dans la continuité avec trois des quatre modèles. Il confirme leur pertinence pour les juges dans le contexte actuel. Le modèle du juge des gens ordinaires ou dévoué est le seul à introduire une relative nouveauté. Certaines des pratiques qui y sont associées, telle la diffusion du droit à la campagne, ont cours depuis plusieurs décennies. Toutefois, l'exigence de contacts directs et proches avec les parties n'a pas toujours été ${ }^{152}$.

Le concours illustre la préférence continue de la CPS pour la médiation judiciaire comme grande orientation pour traiter les conflits. Toutefois, les procès, bien que moins valorisés officiellement, demeurent très présents dans le paysage

150 Heimer, «Cadre responsibility », 129

151 Voir la "Directive n ${ }^{\circ} 9$ ". Comité central du Bureau Général du Parti Communiste Chinois "Communiqué on the current state of the ideological sphere », ChinaFile, (2013) https://www. chinafile.com/document-9-chinafile-translation (consulté le 4 octobre 2018).

152 Zhang, « Judicial reform in China », 21. 
juridique chinois ${ }^{153}$. En outre, telle que pratiquée en Chine, la médiation suscite maintes critiques associées à l'idée d'une harmonie imposée par le juge plutôt qu'à un ordre négocié au sens de Le Roy ${ }^{154}$.

La place des règles juridiques est peu apparente dans les dossiers. Cela est conforme au NMP qui valorise les processus et la flexibilité plutôt que les règles juridiques. En même temps, cette absence de référence au droit est compatible avec la forme du récit, de la pièce de théâtre ou de l'opéra chinois, dont le public saura dégager les passages importants et la morale. Enfin, elle est en accord avec une longue pratique judiciaire qui a historiquement privilégié un résultat perçu comme juste par les parties plutôt qu'une adhésion rigide au formalisme judiciaire ${ }^{155}$. Celle-ci est illustrée par l'emploi de l'adage heqing, heli, hefa ${ }^{156}$. Il en découle un juge de paix qui rend justice en fonction d'une justice particulariste, fondée sur la prise en compte des circonstances individuelles des parties, plutôt qu'un juge professionnel qui fonde sa décision sur une norme générale et impersonnelle (NGI) comme la règle de droit ${ }^{157}$. En outre, le recours à cet adage s'inscrit dans la ligne d'action prônée par les autorités judiciaires chinoises, soit de fonder la résolution des conflits sur les ressources juridiques locales en tenant compte des réalités chinoises ${ }^{158}$. Cette logique identitaire sous-tend le concours dont l'objectif est de faire émerger des modèles aux "caractéristiques chinoises » ${ }^{159}$. L'expression code désigne l'appel à des modèles chinois, par opposition aux modèles présentés comme venant d'Amérique du Nord ou d'Europe. Dans cette optique, le « bon juge » à la chinoise cultive non pas la distance, mais des relations de proximité avec les parties, allant jusqu'aux contacts ex parte avec celles-ci. Il est très éloigné du modèle du juge impartial tel que conçu en Europe et dans le monde angloaméricain $^{160}$. Toutefois, la référence au juge professionnel et impartial apparaît également dans un dossier, celui du juge Chen à Beijing. Cela est conforme aux orientations récentes des autorités, depuis le $14^{\mathrm{e}}$ Plénum du PCC, qui illustrent un retour relatif vers un certain degré de professionnalisation des juges après une phase de populisme judiciaire relevée par Liebman ${ }^{161}$. Le concours suggère une tension continue entre ces deux visions du juge.

Les juges modèles d'une nouvelle ère, héros des récits, ont apparemment la part belle. Isolément ou pris dans leur ensemble, ils donnent de la face à la justice

Ibid., 27.

Le Roy, Le jeu des lois, 152-155. Pour les critiques voir : Minzner, "China's turn against the law »; Woo, "Dynamism of China's civil litigation system ", 148; Ng et He, Embedded courts, 49. Fu « Social harmony », 179-180

Ibid.

157 Sur la NGI, voir Le Roy, Le jeu des lois, 256. Sur l'application impersonnelle du droit, voir : Jeffrey M. Shaman, "The impartial judge: Detachment or passion », De Paul Law Review, 45, n 3 (1996), 605-632, 607.

158 Woo, «Law's Location in China's countryside ». 330.

159 Zhongguo Fayuan Wang (Réseau chinois des cours), « Le bon juge que j'aime le plus ». https:// www.chinacourt.org/article/subjectdetail/id/MzAwNEjPNYABAA\%3D\%3D.shtm (consulté le 20 janvier 2019).

160 Voir, entre autres : Shaman, «The impartial judge », 625; Dominique Noëlle Commaret, « Une juste distance ou réflexions sur l'impartialité du magistrat », Recueil Dalloz, Chronique (1998), 262-264.

161 Liebman, "Populist legality? ", 165; Sur le retour du professionnalisme, voir Zhang, "Judicial reform », 25. 
chinoise comprise comme une institution avec ses acteurs : intègres, durs à la tâche, efficaces, agents de rétablissement de l'harmonie au sein des familles et de la société. Derrière ce portrait élogieux, qui ne peut que réhabiliter l'image de la justice qui préoccupe la Cour, se cachent pourtant de redoutables exigences, parfois contradictoires. Le caractère absolu attaché par le PCC à l'objectif du maintien de la stabilité sociale transforme les cours et les juges en acteurs contraints de pacification. Comment concilier l'efficacité, qui suppose la rapidité dans le traitement des affaires, avec celle de pacifier les parties qui mobilise un temps considérable pour les contacts avec elles? Les juges chinois sont ainsi placés dans un dilemme impossible. Les quatre modèles, loin d'agir comme facteurs de recrutement, risquent plutôt d'aggraver l'exode des juges en cours depuis 2015.

Les slogans de justice des juges sont très importants, comme éléments contextuels, pour la compréhension des modèles de juges. Ils livrent les clés du récit et permettent de dégager les qualités recherchées. En somme, les concours sont de précieux outils de gestion pour la CPS. Instruments de réaction rapide face aux impératifs politiques changeants, ils illustrent également le fait que l'office du juge chinois ne revêt aucunement la stabilité tant valorisée par le PCC. À l'instar des acteurs d'opéra chinois, qui changent de masques avec la rapidité de l'éclair afin d'assumer un nouveau rôle, les juges chinois doivent savoir s'adapter aux mutations fréquentes du contexte politique ${ }^{162}$. Cette qualité, non formulée dans les divers instruments juridiques de la CPS, est sans doute la plus importante pour les juges dans le contexte actuel.

Hélène Piquet

Professeure de droit, sinologue

Membre du Barreau du Québec

Sciences Juridiques, Université du Québec a Montréal

piquet.helene@uqam.ca

162 L'art de changer les masques en une fraction de seconde est une pratique ancienne en Chine, associée à l'opéra de la province du Sichuan mais pratiquée ailleurs également. Siu Wan et Peter Lovrick, Chinese Opera. Images and Stories (Vancouver : UBC Press, 1997), 19. 